\title{
A New Current to The Armamentarium: Is the CHA2DS2-Vasc-HS Score Predictive of Low Left Internal Mammary Artery (LIMA) Flow in Patients Underwent Coronary Bypass Surgery?
}

\author{
Nihat Söylemez, ${ }^{1}$ Mehmet Ball,${ }^{1}$ Fatma Köksal, ${ }^{1}$ Mahmut Yılmaz, ${ }^{1}$ Fazilet Ertürk Sağ,${ }^{1}$ Esra Ertürk Tekin, ${ }^{2}$ \\ Mehmet Ali Yeşiltaş, ${ }^{3}$ Özden Vezir, ${ }^{2}$ Bahar Aydınlı, ${ }^{4}$ Buğra Özkan ${ }^{5}$ \\ ${ }^{1}$ Department of Cardiology, Mersin City Training and Research Hospital, Mersin, Turkey; \\ ${ }^{2}$ Department of Cardiovascular Surgery, Mersin City Training and Research Hospital, Mersin, Turkey; \\ ${ }^{3}$ Department of Cardiovascular Surgery, Bakırköy Dr. Sadi Konuk Training and Research Hospital, İstanbul, Turkey; \\ ${ }^{4}$ Department of Anesthesia and Reanimation, Mersin City Training and Research Hospital, Mersin, Turkey; \\ ${ }^{5}$ Cardiology Clinic, Mersin University Medicine Faculty Mersin, Turkey
}

\section{ABSTRACT}

Introduction: Left internal mammary artery (LIMA) grafts should be used in patients undergoing CABG. No other procedure results in patency equivalent to that of the left anterior descending coronary artery (LAD)-LIMA bypass graft. The CHA2DS2-Vasc-HS scoring system can be used to successfully predict CAD severity in stable CAD patients. We aimed to investigate the relationship between LIMA flow and the CHA2DS2-Vasc-HS score.

Methods: A total of 684 patients, who underwent CABG surgery, were included in this study. Previous history of bypass surgery, emergency operations, patients with Leriche syndrome and patients with severe obstructive pulmonary and subclavian artery disease were excluded from our study. Patients with a LIMA flow that was suitable for bypass grafting, as determined during the intraoperative evaluation, were included in the low LIMA flow group, and the CHA2DS2Vasc-HS score was calculated for all patients.

Results: Patients in the low LIMA flow group (Group 1) were older. The CHA2DS2-Vasc-HS score $(P<0.001)$, presence of mild or moderate COPD $(P=0.022)$, number of severely diseased vessels $(P=0.036)$, and BMI $(P<0.001)$ were independent predictors of poor LIMA flow. The cutoff value of the CHA2DS2-VASc-HS score for the prediction of poor LIMA flow was $>5.5$, with a sensitivity of $92.9 \%$ and specificity of $83.4 \%$ (AUC: $0.938,95 \%$ Cl: $0.906-0.970, P<0.001$ ).

Conclusion: A preoperative high CHA2DS2-Vasc-HS score can be used to predict low intraoperative LIMA flow. The CHA2DS2-Vasc-HS score is an easy-to-use and reliable

Received March 28, 2021; accepted May 10, 2021.

Correspondence: Mebmet Ali YESILTAS, Consultant, Department of Cardiovascular Surgery, S.B.Ü.Bakırköy Dr.Sadi Konuk E.A.H., Zuburatbaba Mah. Dr.Tevfik Sağlam Cad., No: 11, Telephone +90 212-4147171, Fax +90 (212) 4146494 (e-mail: drmaliyes@gmail.com). estimation method and can be used as an additional preoperative of LIMA flow in patients undergoing CABG due to severe CAD.

\section{INTRODUCTION}

Coronary artery bypass grafting (CABG) is the mainstay revascularization option in obstructive coronary artery disease (CAD) patients, especially patients with moderate-high syntax scores, multivessel CAD, and diabetes mellitus (DM) [Neumann 2019]. Conduit selection is the most important aspect of CABG surgery, and the ability to use the left internal mammary artery (LIMA) for left anterior descending artery (LAD) revascularization is the key determinant of positive outcomes [Cohn 2001]. Many studies have shown that the LIMA conduit is superior to other arterial and venous conduits due to its anatomical, structural and physical properties, such as rich endothelial nitric oxide synthase activity, fewer endothelial fenestrations, and lower atherosclerotic burden [Otsuka 2013; He 2011]. Despite these important advantages, risk factors, including lipoprotein levels, DM, smoking habit and advanced age, have been shown to negatively affect LIMA flow and long-term graft patency [Harskamp 2016].

Despite the advantages of medical and interventional therapy options, atherosclerotic cardiovascular disease remains an unsolved problem in daily medical practice. DM, hypertension (HT), hyperlipidemia (HPL), male sex, smoking habit and sedentary lifestyle have been identified as major risk factors for atherosclerotic cardiovascular disease [Perk 2012]. We previously described the CHA2DS2-Vasc-HS [(Congestive heart failure $(\mathrm{C})$, HT $(\mathrm{H})$, Age $\geq 75$ years (A2), DM (D), and Stroke history or TIA (S2), Vascular disease (V), Age 65-74 years (A), and Male gender (as the Sex category), Hyperlipidemia $(\mathrm{H})$, and Smoking $(\mathrm{S})]$ score, and this scoring system was found to successfully predict CAD severity in stable CAD patients [Cetin 2014]. Studies also have shown that the CHA2DS2-Vasc-HS scoring system could be used to predict major cardiovascular events in hospitalized acute coronary syndrome patients and in patients with 
stent restenosis [Zhao 2019; Tasolar 2016]. In addition to these patient groups, patients who undergo CABG and are scheduled to undergo LAD revascularization using the LIMA are other important groups. The identification of relatively high-risk subgroups of CABG patients may be an appropriate approach regarding low LIMA flow status. To our knowledge, no preoperative noninvasive LIMA flow assessment methods have been described. Therefore, we aimed to investigate the relationship between LIMA flow status and the CHA2DS2Vasc-HS score in the perioperative period.

\section{MATERIALS AND METHODS}

Between January 2019 and June 2020, 684 patients who underwent first-time CABG surgery were included in this study. Clinical, demographic and operative data, such as age, sex, DM, HT, HL, smoking status, heart failure (HF), history of ischemic stroke or transient ischemic attack (TIA), and history of peripheral artery disease (PAD), were obtained from patients' files and hospital databases. Patients who underwent emergency CABG surgery, had been diagnosed with acute coronary syndrome in the past two weeks, were scheduled for additional valve or aortic surgery and had undergone previous thoracic surgery were excluded from our study. We also excluded patients with hemodynamic instability, Leriche syndrome, severe obstructive pulmonary and subclavian artery disease, morbid obesity (body mass index > $40 \mathrm{~kg} / \mathrm{m} 2$ ) and a history of radiotherapy to the thoracic region. Informed consent was obtained from all patients, and the study also was approved by our local ethics committee.

In our clinics, all coronary angiography procedures are performed by experienced invasive cardiologists and evaluated by a cardiac team composed of at least two cardiologists and cardiac surgeons. After these evaluations, treatment options, such as medical treatments, percutaneous coronary intervention or CABG surgery, are taken into consideration based on the patient's cardiac and extracardiac characteristics and preferences [Duncan 2011]. In the present study, all CABG surgery decisions were made by the cardiac team, according to the relevant guidelines based on patient characteristics. Smoking was defined as previously described. The presence of any degree of PAD and carotid, vertebral, and subclavian artery disease were accepted as vascular disease and scored. Severe PAD was diagnosed by duplex sonogram and was defined as $>50 \%$ stenosis in the noncoronary arterial systems, such as the carotid vertebral and subclavian arteries. A history of stroke or TIA was obtained from patient records and included in the score calculations. LVEF was calculated using the modified Simpson method, and HF was defined as impaired left ventricular ejection fraction (LVEF <40\%) (M1). HT, HPL, and DM were defined according to current standards [Grundy 2004; Williams 2018]. The CHA2DS2Vasc-HS score was evaluated by two experienced cardiologists, who were blinded to the study design. The CHA2DS2Vasc-HS score was calculated by giving one point each for the presence of HF, HT, DM, vascular disease, age 65-74 years, male sex (as a sex category), HL, and smoking (S) and by adding two points for a history of stroke or TIA and age $\geq 75$ years.

Perioperative management of the study patients was performed by experienced anesthetists, surgeons, and cardiologists. After general anesthesia and intubation, standardized median sternotomy was performed on all patients. Because the use of the LIMA was the preferred method for LAD revascularization in our clinic, LIMA harvesting was performed on all CABG patients according as previously described [Cheng 2015]. Patients whose LIMAs were surgically injured during LIMA harvesting (dissection, crush, etc.) also were excluded from our study. Although suitable LIMA harvesting methods and medical interventions, such as topical papaverine administration, were employed, if our surgeons intraoperatively decided that the LIMA had a low flow, saphenous vein graft (SVG) to LAD revascularization was performed. These patients were included in the low LIMA flow group (Group 1).

Statistics: All analyses were performed using SPSS version 21.0 for Windows (SPSS Inc., Chicago, IL, USA). Numerical variables are presented as means \pm St. deviations, and nominal variables are presented as percentages. All variables were subjected to the Kolmogorov-Smirnov test to determine whether they were normally distributed. The independent samples t-test or Mann-Whitney $U$ test was used to compare the values of continuous variables between the two groups. To evaluate the effects of factors on low LIMA flow, we performed multivariate logistic regression analyses. The model was adjusted using various candidate factors, such as ACE usage, statin usage, number of grafted vessels, concomitant valve disorders, body surface area, presence of $\mathrm{AF}$ and CHA2DS2-Vasc-HS score. A receiver operator characteristic (ROC) curve was constructed to determine the predictive value of the CHA2DS2-Vasc-HS score for low LIMA flow. Coefficients with $95 \%$ confidence intervals (CIs) are presented. A $P$-value $<0.05$ was considered significant.

\section{RESULTS}

A total of 684 patients, who underwent CABG surgery, were included in our study, and patients were divided into two groups, according to LIMA flow status (Group 1: low LIMA flow, $N=28$ and Group 2: adequate LIMA flow, $N=656$ ). Patients with low LIMA flow were older. The low LIMA flow group also had higher proportions of patients with HPL, smoking, PAD, stroke history, HF, and COPD $(P<0.001$ for all). When we evaluated past medical history, ACEI usage was more common and statin usage was less common in group 1 ( $P=0.029$ and $P=0.026$, respectively). The use of other medical therapies, such as beta-blockers (BBs) and calcium channel blockers (CCBs), were found to be similar between the two groups. Because patients with severe valve disorders were excluded from our study, we compared mild or moderate aortic and mitral valve disorders between the two groups, and we found no difference between the two groups. We also evaluated patients' operative characteristics, and the number of severely diseased vessels, proportion of patients with SVG to diagonal branch revascularization and proportion of patients 
Table 1. Clinical, demographic and operative characteristics of patient groups, according to LIMA flow

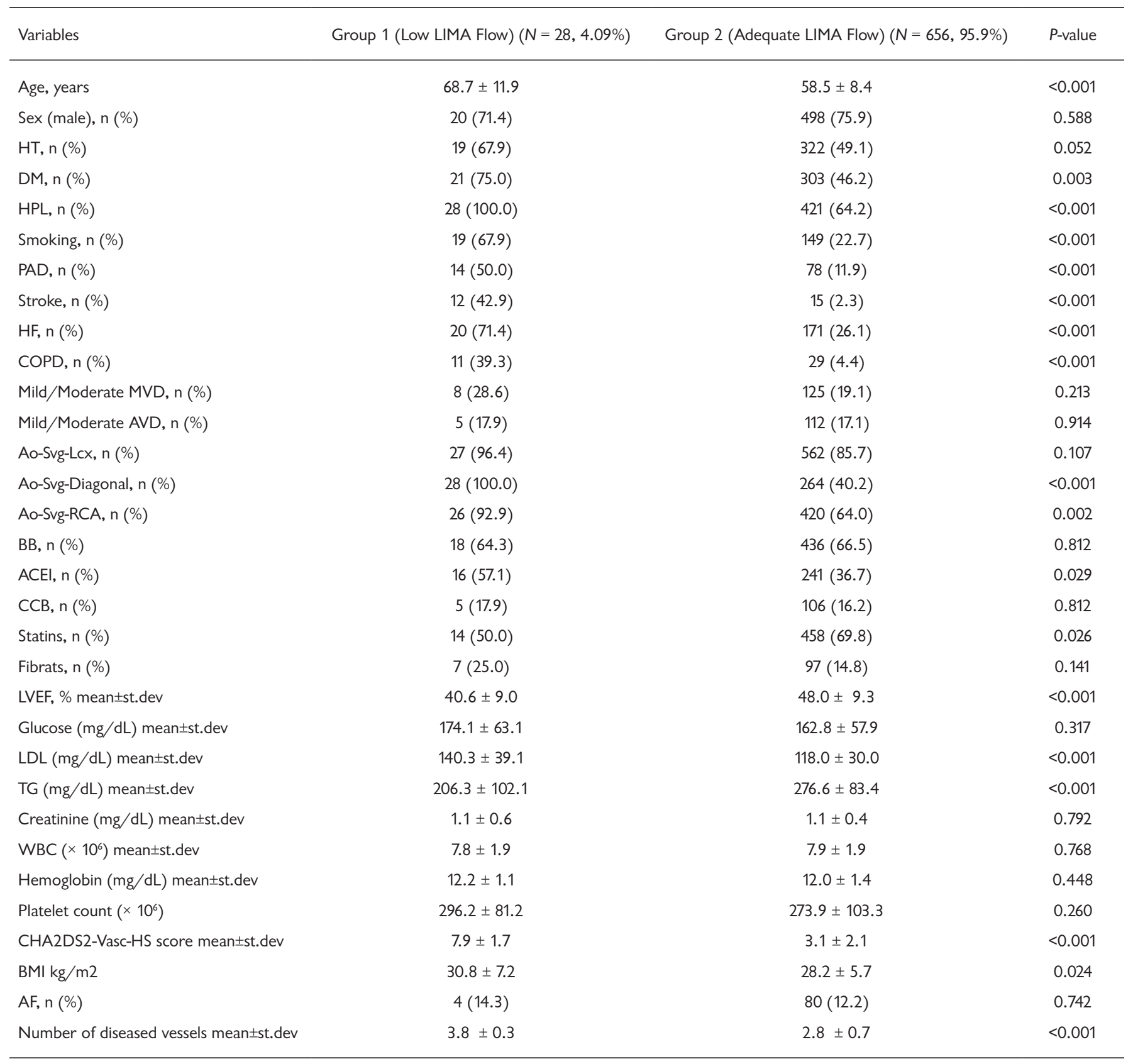

HT, hypertension; DM, diabetes mellitus; HPL, hyperlipidemia; PAD, peripheral artery disease; HF, heart failure; COPD, chronic obstructive pulmonary disease; MVD, mitral valve disease; AVD, aortic valve disease; Ao, aorta; Svg, saphenous vein graft; Lcx, left circumflex artery; RCA, right coronary artery; BB, beta blocker; ACEI, angiotensin-converting enzyme inhibitor; CCB, calcium channel blocker; LVEF, left ventricular ejection fraction; LDL, low-density lipoprotein; TG, triglyceride; WBC, white blood cell; BMI, body mass index; $\mathrm{AF}$, atrial fibrillation

with SVG to RCA revascularization were higher in the low LIMA flow group $(P<0.001, P<0.001$ and $P=0.002$, respectively). In the present study, CHA2DS2-Vasc-HS scores were calculated by two experienced cardiologists who were blinded to the study design. Inter- and intraobserver variations for the scoring system were found to be $2.9 \%$ and $3.1 \%$, respectively.
CHA2DS2-Vasc-HS scores were compared between the two groups, and Group 1 patients had higher CHA2DS2-VascHS scores than Group 2 patients $(P<0.001)$. Other clinical and laboratory characteristics of the study groups are shown in Table 1. (Table 1)

To determine the predictors of low LIMA flow status, 
Table 2. Predictors of low LIMA flow

\begin{tabular}{lcccc}
\hline Variables & B & OR & $95 \% \mathrm{Cl}$ & P-value \\
\hline CHA2DS2-Vasc-HS & 0.854 & 2.349 & $1.608-3.432$ & $<0.001$ \\
Statins & -1.658 & 0.191 & $0.056-0.650$ & 0.008 \\
BMI kg/m2 & 0.194 & 1.215 & $1.089-1.354$ & $<0.001$ \\
Number of severely diseased vessels & 1.578 & 4.843 & $1.109-21.158$ & 0.036 \\
COPD & 1.383 & 3.986 & $1.219-13.034$ & 0.022 \\
\hline
\end{tabular}

COPD, chronic obstructive pulmonary disease; BMI, body mass index

multivariate regression analyses were performed using a fully adjusted model, including candidate factors (such as COPD, operative characteristics, BMI, presence of AF, and ACEI and statin usage) that could contribute to poor LIMA flow. Other probable candidate factors that represented the CHA2DS2Vasc-HS scoring system were not included separately in this analysis. The CHA2DS2-Vasc-HS score $(P<0.001)$, presence of mild or moderate COPD $(P=0.022)$, number of severely diseased vessels $(P=0.036)$, and BMI $(\mathrm{P}<0.001)$ were independent predictors of low LIMA flow. We also found that the lack of the use of statins independently predicted low LIMA flow. (Table 2) To detect the optimal cutoff value for the CHA2DS2-VASc-HS score, an ROC curve was constructed. The cutoff value of the CHA2DS2-VASc-HS score for the prediction of low LIMA flow was $>5.5$, with a sensitivity of 92.9\% and specificity of $83.4 \%$ (AUC: $0.938,95 \%$ CI: 0.906 $-0.970, P<0.001$ ). (Figure 1 )

\section{DISCUSSION}

Our study is the first to evaluate the relationship between the CHA2DS2-Vasc-HS score and low LIMA flow in patients undergoing CABG. We found that a higher CHA2DS2-VascHS score predicted a low intraoperative LIMA flow. In addition to this scoring system, our study showed that the number of grafted vessels, presence of COPD, and higher BMI independently predicted perioperative low LIMA flow. We also found that statin use had a positive impact on the LIMA flow. As the preferred conduit option, LIMA has been used for LAD grafting for many years, and it is well known that LIMA grafts are more durable and have higher patency rates than other conduit options in patients with diffuse coronary artery disease [Haberal 2015]. In our study, LAD to LIMA grafting was not performed in $28(4.09 \%)$ patients who underwent the CABG procedure due to low LIMA flow.

Although there has been an increasing trend in the use of LIMA for LAD revascularization in recent years, studies have reported variable LIMA use rates. Karthik et al. showed that $8.2 \%$ of patients, who underwent CABG, did not undergo LIMA to LAD revascularization, and they also found that elderly patients; patients with DM, HF, COPD, poor LIMA flow, and a history of stroke; patients undergoing additional valve surgery; and patients needing emergency surgery were

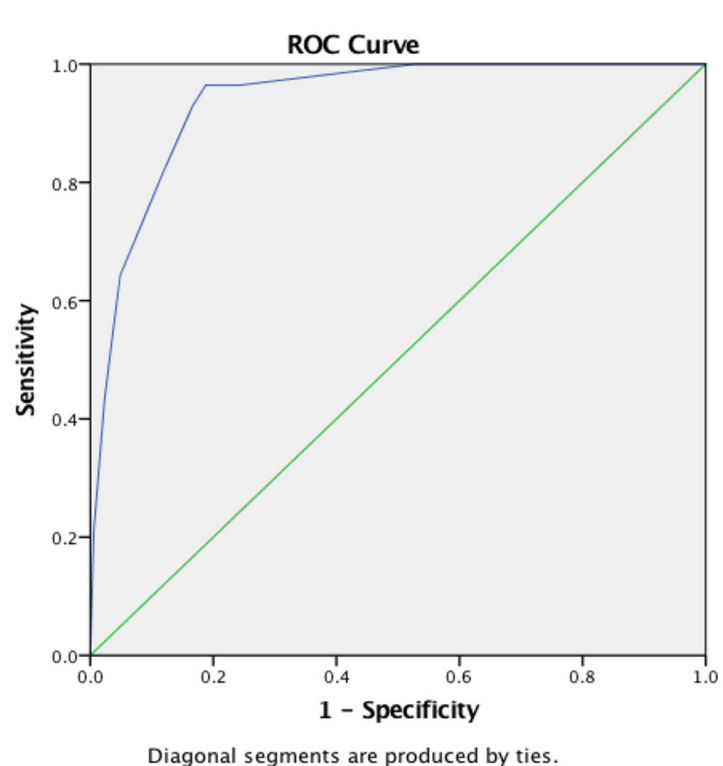

Figure 1. ROC curve analysis of low LIMA flow and CHA2DS2-Vasc HS score.

$\begin{array}{ccccc}\text { CHA2DS2-Vasc HS } & \text { AUC } & \text { SE } & 95 \% \mathrm{Cl} & P \text { Value } \\ & 0.938 & 0.017 & 0.906-0.970 & <0.001\end{array}$

at high risk for LIMA nonusage [Karthik 2004]. Similar to Karthik et al., we found that patients with DM, HF, stroke, and older age were at high risk for LIMA nonusage, and the CHA2DS2-Vasc-HS scoring system includes all of these parameters. In our study, the proportion of patients in whom LIMA could not be used, due to low flow, was relatively high. The smaller number of study patients and the definition of low LIMA flow based on the discretion of the surgeon in the perioperative period may help explain this difference. Previous revascularization guidelines recommended graft flow assessment, using relatively objective tools, such as the mean graft flow and pulsatility index [Kolh 2010]. However, the optimal technique and cutoff values for these methods have not clearly been identified. Some operators recommended not using the LIMA if its flow is $20<\mathrm{mL} / \mathrm{min}$; however, others suggested using the LIMA if the pulsatility was suitable even if the flow was low [Honda 2015; Hata 2000]. Because of these debates 
and technical and financial concerns, most low LIMA flow decisions are relatively subjective in daily practice, based on the opinion of the surgeons in the perioperative period.

The CHA2DS2-Vasc-HS scoring system includes most modifiable and nonmodifiable atherosclerotic risk factors, such as HT, age, DM, HPL, smoking, and PAD. Studies have shown that this scoring system can be used to predict CAD severity and complexity in patients with stable CAD and non-STelevation acute coronary syndrome [Cetin 2014; Tasolar 2016]. (D3-4). Similar to these findings, our study results showed that patients with low LIMA flow had more severely diseased coronary vessels and higher CHA2DS2-Vasc-HS scores. LIMA has higher endothelial nitric oxide synthase levels and is prone to less atherosclerosis [Otsuka 2013]. One study showed that patients with $\mathrm{DM}$ and $\mathrm{CAD}$ had subclinical endothelial changes in the LIMA even if the LIMA was free from gross atherosclerotic changes [Bahar 2020]. In addition, atherosclerosis is a common disorder that can affect the circulation in most arteries, including the LIMA, and the relationship between atherosclerotic risk factors and endothelial dysfunction is well known. Therefore, subclinical endothelial dysfunction in patients with higher CHA2DS2-Vasc-HS scores might have been related to low LIMA flow in our study group.

In addition to the CHA2DS2-Vasc-HS score, a higher $\mathrm{BMI}$ and the presence of COPD were found to be predictors of low LIMA flow and the consequent decision not to use the LIMA. Similar to the present study, Leavitt et al. showed that obese patients were less likely to undergo revascularization with the LIMA [Leavitt 1997]. However, they could not clearly explain this outcome. In our study, because our patients with higher BMI values were more likely to have other atherosclerotic risk factors, such as DM, HT, and HPL, these patients may be more affected by endothelial dysfunction, low-grade inflammation, and impaired LIMA flow [Grover-Páez 2009; Lambert 1996; Van Guilder 2006]. It is not surprising that the lack of the use of statins also was found to be an independent predictor of low LIMA flow and that the proportion of patients using statins was higher in the adequate LIMA flow group. Studies have shown that statin use decreases atherosclerotic plaque volume, increases plaque stability, and reduces the major cardiovascular event rate [Wojszel 2019]. As lipid-lowering agents, statins also have positive effects on endothelial function. In our study groups, the adequate LIMA flow status in group 2 could be the result of the positive anti-inflammatory effects of statins on endothelial function [Davignon 2004; Oesterle 2017].

\section{CONCLUSION}

Patients who undergo CABG surgery due to severe CAD have more than one risk factor and comorbidities. Therefore, simple risk prediction models, such as the CHA2DS2Vasc-HS score, may facilitate the achievement of optimal CABG procedure results. Based on the results of this study, a high CHA2DS2-Vasc-HS score can predict low LIMA flow. Therefore, objective assessments of LIMA flow may be needed either preoperatively or intraoperatively in patients with high CHA2DS2-Vasc-HS scores. Furthermore, because most of the parameters that have been included in the scoring system, such as HF, stroke, HPL and smoking, are modifiable risk factors, eliminating these factors in patients planning to undergo CABG who are at risk of low LIMA flow might also be reasonable. Despite these important results, our study has some limitations. First, our surgeons evaluated the LIMA flow subjectively, and we could not evaluate endothelial dysfunction due to the retrospective design. Second, the present study was a single-center study, and the relatively small number of patients may have affected our results.

\section{REFERENCES}

Bahar L, Tüysüz ME. 2020. The Relationof Left Internal Mammary Artery Atherosclerosis With Urotensin II. Bratislava Medical Journal. 121: 516-521.

Cetin M, Cakici M, Zencir C, et al. 2014. Prediction of coronary artery disease severity using CHADS2 and CHA2DS2-VASc scores and a newly defined CHA2DS2-VASc-HS score. Am J Cardiol. 15; :950-6.

Cheng K, Rehman SM, Taggart DP. 2015. A Review of Differing Techniques of Mammary Artery Harvesting on Sternal Perfusion: Time for a Randomized Study? Ann. Thorac. Surg. 100: 1942-53.

Cohn LH. 2001. Use of Internal Mammary Artery graft and in-hospital mortality and other adverse outcomes associated with coronary artery bypass surgery. Circulation. 103: 483-4.

Davignon J. 2004. Beneficial cardiovascular pleiotropic effects of statins. Circulation. 109: 39-43.

Duncan RF, Dundon BK, Nelson AJ, et al. 2011. A study of the 16-Segment Regional Wall Motion Scoring Index and biplane Simpson's rule for the calculation of left ventricular ejection fraction: a comparison with cardiac magnetic resonance imaging. Echocardiography 28: 597-604.

Grover-Páez F, Zavalza-Gómez AB. 2009. Endothelial dysfunction and cardiovascular risk

factors. Diabetes Res Clin Pract. 84: 1-10.

Grundy SM, Cleeman JI, Merz CN, et al. 2004. National Heart, Lung, and Blood Institute; American College of Cardiology Foundation; American Heart Association. Implications of recent clinical trials for the National Cholesterol Education Program Adult Treatment Panel III guidelines. Circulation. 110: 227-39.

Haberal I, Gurer O, Ozsoy D, Erturk E. 2015. Coronary flow reserve in patients with left anterior descending artery-left internal mammary artery long patch plasty anastomosis: a prospective study. J Cardiothorac Surg. 10: 51.

Harskamp RE, Alexander JH, Ferguson TB, Hager R, Mack MJ, Englum B. 2016. Frequency and Predictors of Internal Mammary Artery Graft Failure and Subsequent Clinical Outcomes: Insights From the PREVENT IV Trial. Circulation. 12; 131- 138.

Hata M, Shiono M, Orime Y, et al. 2000. Clinical results of coronary artery by pass grafting with use of internal thoracic artery under low free flow conditions.J.Thorac Cardiovas Surg 119: 125-9.

He GW, Fan L, Grove KL, Funnary A, et al. 2011. Expression and function of endothelial nitric oxide synthase messenger RNA and protein are higher in internal mammary than in radial arteries. Ann Thorac Surg. 92; 845-850. 
Honda K, Okamura Y, Nishimura Y, et al. 2015. Graft flow assessment using a transit time flow meter in fractional flow reserve-guided coronary artery bypass surgery. J Thorac Cardiovasc Surg. 149: 1622-8.

Karthik S, Srinivasan AK, Grayson AD, Jackson M, Mediratta NK. 2004. Left Internal Mammary Artery to the Left Anterior Descending Artery: effect on morbidity and mortality and reasons for nonusage. Ann Thorac Surg. 78: 142-8.

Kolh P, Wijns W, Danchin N, et al. 2010. Guidelines on myocardial revascularization. Task Force on Myocardial Revascularization of the European Society of Cardiology (ESC) and the European Association for Cardio-Thoracic Surgery (EACTS); European Association for Percutaneous Cardiovascular Interventions (EAPCI). Eur J Cardiothorac Surg. 38(Suppl): S1-S52.

Lambert J, Aarsen M, Donker AJ, Stehouwer CD. 1996. Endotheliumdependent and-independent vasodilation of large arteries in normoalbuminuric insulin-dependent diabetes mellitus. Arterioscler Thromb Vasc Biol. 16: 705-11.

Leavitt BJ, Olmstead EM, Plume SK, et al. 1997. Use of the Internal Mammary Artery Graft in Northern New England. Circulation. 96 (Suppl II): 32-7.

Neumann FJ, Sousa-Uva M, Ahlsson A, et al. 2019. 2018 ESC/EACTS Guidelines on myocardial revascularization. The Task Force on myocardial revascularization of the European Society of Cardiology (ESC) and European Association for Cardio-Thoracic Surgery (EACTS). European Heart Journal. 40: 87-165.

Oesterle A, Laufs U, Liao JK. 2017. Pleiotropic effects of statins on the cardiovascular system. Circ Res. 120: 229-243.

Otsuka F, Yahagi K, Sakakura K, Virmani R. 2013. Why is the mammary artery so special and What protect it from atherosclerosis? Ann Cardiothorac Surg. 2: 519-526.
Perk J, De Backer G, Gohlke H, et al. 2012. European Association for Cardiovascular Prevention \& Rehabilitation (EACPR); ESC Committee for Practice Guidelines (CPG). European Guidelines on cardiovascular disease prevention in clinical practice (version 2012). The Fifth Joint Task Force of the European Society of Cardiology and Other Societies on Cardiovascular Disease Prevention in Clinical Practice (constituted by representatives of nine societies and by invited experts). Eur Heart J. 33; 1635-701.

Tasolar H, Cetin M, Balli M, et al. 2016. CHA2DS2-Vasc-HS score in non-ST elevation acute coronary syndrome patients: assessment of coronary artery disease severity and complexity and comparison to other scoring systems in the prediction of in-hospital major adverse cardiovascular events. Anatol J Cardiol. 16: 742-8.

Van Guilder GP, Hoetzer GL, Dengel DR, Stauffer BL, DeSouza CA. 2006. Impaired endothelium- dependent vasodilation in normotensive and normoglycemic obese adult humans. J Cardiovasc Pharmacol. 47: 310-3.

Williams B, Mancia G, Spiering W, et al. 2018. 2018 ESC/ESH Guidelines for the management of arterial hypertension: The Task Force for the management of arterial hypertension of the European Society of Cardiology (ESC) and the European Society of Hypertension (ESH). European Heart Journal. 33: 3021-3104.

Wojszel ZB, Kasiukiewicz A, Swietek M, Swietek ML, Magnuszewski L. 2019. CHA2DS2-VASc score can guide the screening of atrial fibrillation - cross-sectional study in a geriatric ward. Clinical Interventions in Aging. 14: 879-887.

Zhao SG, Xu JJ, Tao ZH, et al. 2019. CHA2DS2-Vasc score and CHA2DS2-Vasc-HS score are poor predictors of in-stent restenosis among patients with coronary drug-eluting stents. Journal of International Medical Research. 47; 2533-2544. 